(2) OPEN ACCESS

\title{
Is informed consent required for the diagnosis of brain death regardless of consent for organ donation?
}

\author{
Osamu Muramoto
}

Correspondence to

Dr Osamu Muramoto, Center for

Ethics in Health Care, Oregon

Health and Science University,

Portland, OR 97239, USA;

muramoto@ohsu.edu

Received 26 March 2020

Revised 6 May 2020

Accepted 11 May 2020

Published Online First

5 June 2020

\section{ABSTRACT}

In the half-century history of clinical practice of diagnosing brain death, informed consent has seldom been considered until very recently. Like many other medical diagnoses and ordinary death pronouncements, it has been taken for granted for decades that brain death is diagnosed and death is declared without consideration of the patient's advance directives or family's wishes. This essay examines the pros and cons of using informed consent before the diagnosis of brain death from an ethical point of view. As shared decision-making in clinical practice became increasingly indispensable, respect for the patients' autonomous wishes regarding how to end their lives has a significant role in deciding how death is diagnosed. Brain death, as a fully technologically controlled death, may require a different ethical framework from the old one for traditional cardiac death. With emerging and proliferating options in end-of-life care for those who suffer from catastrophic brain injury, the traditional reasoning that 'death gives no choice, hence no consent' requires another examination. Patients facing imminent brain death now have options other than undergoing the diagnostic workup for brain death, such as donation after circulatory death and withdrawal of life-sustaining treatment with maximum comfort measures for death with dignity. Nevertheless, just as in the debate over opt-in versus opt-out organ donation policies, informed consent before the diagnosis of brain death faces fierce opposition from consequentialists urging the expansion of the donor pool. This essay examines these objections and provides constructive replies along with a proposal to accommodate this morally required consent.

\section{INTRODUCTION}

Is consent for the diagnosis of brain death (DBD) presumed? At first thought, the obvious answer seems to be yes. Why not? Doctors make all sorts of diagnoses in clinics and hospitals without explicit consent all the time. It is presumed (or implicit) by virtue of the fact that one is under the care of doctors. Why do we need separate consent for the DBD? However, when it comes to morally sensitive diagnoses, the answer is not so straightforward.

Obviously when the diagnostic procedure, such as colonoscopy, involves certain risks, informed consent is standardly offered before the diagnostic workup. Another situation occurs when a diagnosis deeply impacts one's destiny, such as tests for cancer genes, Huntington's disease and HIV infection. However, there is something conceptually odd about consenting to diagnosing death. Does one live longer by refusing the diagnosis of death?

The second part of the question in the title is also puzzling. If consent for organ donation is presumed, one might infer that consent for the entire process of donation from the DBD is also presumed. Indeed, consent for organ donation, whether presumed or explicit, is silent regarding the DBD. For example, in England where presumed (or deemed) consent for donation started in May 2020, there is no reference to DBD or any other antemortem interventions necessary for organ procurement. ${ }^{1-3}$ Nevertheless, you cannot presume that consenting to donation entails consenting to the DBD because a DBD can be made independent of organ donation, and organ donation can be executed without a DBD. A DBD while still alive (antemortem), and organ procurement after death (postmortem) are, morally, entirely separate procedures. Moreover, in jurisdictions with explicit consent for donation such as the USA, consent for donation is usually offered after the DBD.

Issues surrounding consent for the DBD have been seldom discussed in the literature of philosophy, ethics or medicine until very recently. ${ }^{4-10}$ As I review shortly, there are many reasons why this question is quickly dismissed. Yet, what has evolved over the recent several years regarding the contentious issues over brain death (BD) makes us reconsider our tradition of presumed consent for the DBD: whether consent for organ donation should be explicit or presumed; a highly publicised US case involving Jahi McMath, who demonstrated that a DBD properly made by two highly qualified neurologists may still turn out to be false positive (she 'survived' BD for 4 years and evolved into a probable minimally conscious state $)^{11}{ }^{12}$; the increasing use of donation after circulatory determination of death (DCDD) without BD; and whether the nontherapeutic prolongation of dying process before the $\mathrm{DBD}$, or so-called elective ventilation, without consent is morally permissible. ${ }^{13}$

\section{ARGUMENT FOR EXPLICIT CONSENT FOR THE DBD}

The ethical bedrock of informed consent rests on respect for autonomy. An explicit consent enables the patient/surrogate to choose from options including non-intervention. ${ }^{\mathrm{i}}$ The landmark report of the US President's Commission states:

Fundamentally, informed consent is based on respect for the individual, and, in particular, for each individual's capacity and right both to define his or her own goals and to make choices designed to achieve those goals (p 17). ${ }^{14}$

${ }^{\mathrm{i}}$ When the DBD is being contemplated, the patient is always completely unconscious. When I refer to 'patient/surrogate' hereafter, it is shorthand for 'the patient as represented by the surrogate, the family, advanced directives, or other forms of relevant representation previously expressed by the patient'. 
While risks of physical harm are considered at the centre of informed consent in contemporary clinical practice, the impact of a proposed intervention on one's life goals is fundamental to one's self-determination, as underscored in this quote. Undergoing the procedure of DBD appears to have a serious impact on end-of-life (EOL) goals comparable to a do-not-resuscitate order or other measures of foregoing life-sustaining treatment (LST). Physical harms aside (discussed later), the treatment and invasion of a dying body during the diagnostic procedure for BD and thereafter in a brain-dead state might be perceived by some as undignified and objectionable. The public generally desire frank and open discussions on EOL decisions. Studies repeatedly show that compassionate communication and shared decision-making are ranked highest in EOL care. ${ }^{15} 16$

Nevertheless, as the President's Commission also stated, patients' autonomy is by no means absolute. It has to be balanced against other values and principles to guide clinicians to manage the moral challenges of EOL care. The most important competing value in this context is organ procurement to benefit other patients who are critically ill. The argument for presumed consent for organ donation justifies the limitation of explicit consent for this reason. Is the DBD exempt from explicit consent for the same reason?

\section{MULTIPLE CHOICES TO CONSIDER BEFORE BD DETERMINATION}

In the USA, clinicians are required to contact a local organ procurement organisation (OPO) to facilitate organ donation when a potential organ donor is identified. ${ }^{17}$ This contact is triggered by a recognition of imminent brain death (IBD) in a mechanically ventilated patient who has a very low Glasgow Coma Scale score $(<5)$, lost more than three brainstem reflexes and is considered for the DBD or withdrawal of life-sustaining treatment (WLST) due to poor prognosis. At this point, we have at least five options before proceeding to BD testing:

1. DBD followed by organ procurement, if DBD is positive and the patient is an organ donor.

2. DBD followed by WLST with comfort measures, if DBD is positive and the patient is a non-donor.

3. WLST with comfort measures for those whose priority is dignified death over prolonged LST with severe neurological disability. This is also a fallback position of option 2 if DBD is negative.

4. DCDD if the patient is a donor, medically qualified for DCDD and, for various reasons, wants to avoid option 1 or is unqualified for option 1 (including negative DBD), but consents to DCDD.

5. Postpone a decision, continue the best treatment for now and wait for further evolution of the clinical status and family's clear preferences. This implies that the patient may die the next day of cardiac arrest, or improve and come out of IBD, or the family may decide from option 1 to 4 . If IBD continues, this decision process should iterate.

Obviously, not all IBD cases have all five options; they can emerge over time. Thus, most decisions are conditional, hinging on how the clinical status evolves (option 5). Nevertheless, this conceptual scheme shows that we now have at least four combinations, DBD yes/no $\times$ organ donation yes/no. For example, someone who wants to donate but decline the DBD may find option 4 most desirable. Conversely, someone who is committed to donation and BD can eagerly choose option 1. In such cases, non-therapeutic antemortem interventions, such as withholding/ withdrawing measures to reduce intracranial pressure, which could hasten the progression to $\mathrm{BD}$, might be morally acceptable if performed after full disclosure. Yet another family/surrogate may want to continue the best treatment given a meagre possibility of improvement. In such cases, multiple iterations of option 5 may be necessary.

I suggest that the best way to handle this complex choice in the face of extremely poor prognosis is to hold an urgent conference with at least three stakeholders: representatives of the patient (family/surrogate), the intensive care unit (ICU) medical team and a local OPO if the patient is a potential donor. The major concern expressed by OPOs is not to initiate the discussion of organ donation by the treating medical team to avoid conflict of interest. ${ }^{18-20}$ Meanwhile, as policymakers for organ transplantation are under enormous pressure to expand the donor pool, OPOs are also hard pressed not to miss even the slightest chance to recruit one more donor. In order to avoid direct conflict of interest regarding the best interests of the patient vis-à-vis organ procurement, it seems advisable that this conference should be led by a third party. The best person would be a palliative care professional (physician, social worker or nurse), who is trained to compassionately guide the families of patients who are imminently dying. ${ }^{\text {ii }}$ Their primary interest is advocating the patient's dignified, comfortable and fulfilling dying, which includes her wishes, beliefs, values and, most importantly, desire to donate organs.

The most important and useful feature of this conference is that all the EOL care options are disclosed to the family/surrogate at first, and they are given a chance to examine and consent to or decline these. Instead of a unilateral DBD followed by an intense negotiation for organ donation, the family/surrogate has a chance to consent to a certain combination of all those options, namely consent for the DBD, WLST and DCDD, in conjunction with organ donation. The expertise of the palliative care specialist is particularly valuable for families who have difficulty in deciding due to the overwhelming stress of imminent death. Instead of aggravating their mounting psychological stress by pressuring organ donation, a palliative care specialist can provide timely advice for grieving and reassurance for dignified dying.

If time permits, the first meeting should focus on providing a map of all options and answering questions without immediate decision (option 5), which is almost impossible for the overwhelmed family/surrogate. Follow-up meetings would enable them to digest the information and achieve a better informed decision.

However, I do recognise that strong opposition to this approach from different directions exists. In what follows, I consider several important objections. The goal is to further crystallise the points of disagreement, and seek a more ethical, shared and transparent decision process for the DBD.

\section{REPLY TO MAJOR OBJECTIONS}

\section{Philosophical-metaphysical argument}

This objection was briefly mentioned above: Death has never been determined after consent. If the patient/surrogate can decline the diagnosis of death, that refusal cannot alter the fact that the patient is dead. Thus, the consent/decline for death determination is incoherent.

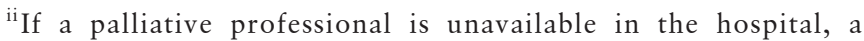
social worker attached to the ICU with necessary training for this role may be a reasonable alternative. The point is that, as recommended by OPOs, it is not the treating clinicians, and at the same time it is not the OPOs themselves, due to conflicts of interest.
} 
It is true that when there is no viable alternative, which is usually no intervention in most medical contexts, consent is meaningless. Since traditional circulatory death (CD) in normal clinical context happens after all life-prolonging measures are exhausted or foregone, there is no alternative left but to proceed to death determination. This is why traditional death determination is outside the scope of consent. In contrast, as discussed above, before undertaking the investigation of the DBD, there are other options to choose from. This is the fundamental difference between traditional death and BD. When options exist, shared decision-making is essential. If BD testing is performed unilaterally, the patient is deprived of a chance to choose WLST that is consistent with their EOL goals, for example.

\section{Legal argument}

Determining and pronouncing death is a legal duty of a physician. This legal decision cannot be refused.

Legal pronouncement of death is important for many nonmedical reasons, such as disposition of the body, insurance claim, public health statistics, and so on. Nevertheless, if we separate the concept of the legal confirmation that death has occurred from one method of medical investigation to see if the patient is in a brain-dead state, one can accept the former but at the same time refuse the latter. There is no legal requirement to use one method over another to confirm death. It is a matter of clinical judgment. In the case of traditional death determination, one may accept, for example, auscultation and ECG to determine asystole, but not opening the chest wall and visually inspecting the heart, even when a stethoscope or an ECG machine is immediately unavailable. This leads to another related legal challenge.

The law does not allow choosing the definitions and criteria of death, $C D$ or $B D$ (except for religious accommodation in the state of New Jersey and non-donors in Japan). ${ }^{21}$ Therefore, refusing BD by refusing the $D B D$ is illegal.

It is understood that death determination consists of three separate conceptual components: definition, criteria and test. ${ }^{22-24}$ This objection conflates the definition and criteria with the test. Consent for the DBD is the consent only for the test, and not for the definition or criteria for BD. Just as declining a colonoscopy does not entail the denial of colon cancer, so too declining the DBD does not entail the rejection of $\mathrm{BD}$ as a definition of death. It is true that $\mathrm{BD}$ can only be diagnosed by the tests for BD. However, death can be diagnosed by other methods that can be accepted. Since a legal death is a unitary phenomenon judged by different methods, declining only one of them, like any other medical tests, should not be prohibited. In fact, some patients with IBD develop cardiac arrest within several hours to days and die before BD testing. Neither failing to start BD testing in time and then diagnosing $\mathrm{CD}$ is illegal, nor is diagnosing $\mathrm{BD}$ by averting the antecedent $\mathrm{CD}$ by resuscitation. These decisions are not mandated by the law, but left for clinical judgment, which allows for shared decision-making as needed.

\section{Administrative argument}

$D B D$ is a necessary medical procedure for clinical management in the ICU, and it is within the realm of general consent for hospital admission. ${ }^{9}$

Many assessments and procedures are performed in busy, fastpaced hospital settings with numerous professionals involved. Another requirement for consent is cumbersome and slows down necessary treatments. Most minor procedures and assessments are covered by the general consent for hospitalisation. This is particularly true in the ICU, where many patients are too sick to give consent and many interventions are considered emergent, which are also covered by presumed consent. Is the DBD one of those procedures?

The answer is clearly negative. There is no direct benefit for the patient who is dying, particularly if the patient's status as an organ donor is unknown and there is harm. ${ }^{8}$ The magnitude of the interest of the patient impacted by this procedure is comparable to WLST and DCDD, which are generally performed with explicit consent in the ICU. Moreover, even if it is covered by the general consent for hospitalisation, nothing prevents the hospital from obtaining explicit consent for a non-therapeutic and risky intervention.

Nevertheless, we are not dealing with any absolute law here. In case of insurmountable difficulty in getting consent, such as when the family/surrogate is unavailable within reasonable time frame, or they decline to participate in decision-making, a more pragmatic approach is warranted.

A related argument from the viewpoint of the ICU operation is as follows: Physicians in the ICU are overwhelmed by an increasing burden of administrative rules and documentation requirements on top of the ever-increasing complexity of medical technologies and protocols. Another informed consent is the last thing they can handle.

A recently published survey shows that the majority of neurologists $(78 \%)$ replied that consent for the DBD is unnecessary. ${ }^{25}$ This result, however, is not surprising. While I am extremely sympathetic to those dedicated and overloaded physicians, this survey is analogous to asking a manufacturer of medical devices if one more safety regulation is necessary. A more unbiased survey should be directed to the general public or the population of patients and their families (consumers), and not the providers of the procedure themselves. Though I am unaware of such a survey, I expect that the public find it reasonable to have one last chance to reflect on EOL decisions, judged by other surveys on EOL care.

As discussed earlier, the best coordinator for this consent process and all EOL decisions would be an expert in palliative care, not intensivists or neurologists. The care conference proposed here would relieve the direct burden on them. However, by sending a representative, their opinions and recommendations are well reflected in the outcome.

\section{Safety argument}

$B D$ testing is safe and therefore informed consent is unnecessary. ${ }^{626}$

Most discussions on informed consent for the DBD focus on the safety issue of the apnea test. ${ }^{568926}$ This test consists of evaluating the brain's response to deliberately raised blood $\mathrm{CO}_{2}$ level. ${ }^{\text {iii }}$ If there is no response by exhibiting respiratory movement driven by the brainstem respiratory centre, the test is judged positive for the loss of lower brainstem function. Though it comprises only one part of BD testing, it is considered essential because other ancillary tests, such as electroencephalogram and imaging demonstrations of the lack of cerebral circulation, are considered less reliable. ${ }^{27}$ Major complications of the apnea test include hypotension, hypoxaemia, arrhythmia and fatal cardiac arrest. The safety of the apnea test is slowly improving; $15 \%-39 \%$ of complications were reported in $2000 .{ }^{28}$ However, the most recent report from the same group showed a marked improvement to $1.7 \%$ ( 2 out of 116 cases). ${ }^{26}$

\footnotetext{
iii Due to space limitation, please refer to Wijdicks ${ }^{46}$ for all tech nical details, and Joffe et $a l^{32}$ for a critical review of the problems of validity, complications and risks of the apnea test.
} 
Nevertheless, it is important to put this complication rate in perspective. The complication rate of colonoscopy is $0.28 \%,{ }^{29}$ and that of an exercise ECG test is $\leq 0.02 \% .^{30}$ Even then, both those diagnostic tests routinely require explicit written consent. Even if the safety of the apnea test has improved significantly, it is not low enough to eliminate explicit consent. ${ }^{8}$ However, a more serious safety concern is the methodological impossibility of tracking down cases of false-positive BD diagnosis (or the problem of self-fulfilling prophecy). ${ }^{10}$ Only through extremely rare cases, such as Jahi McMath, do we know that more uncounted false-positive cases exist. ${ }^{11} 31$ Another concern is the possibility that the test itself is precipitating $\mathrm{BD}$ by raising the intracranial pressure through hypercapnia, in effect, causing its own positive result-another case of self-fulfilling prophecy. This is also methodologically impossible to track down. ${ }^{32}$ Moreover, the requirement for informed consent is much more than simple risk calculation for physical harm. As stated above, a core tenet of informed consent is to provide a chance to decide based on the knowledge of how this test result affects the patient's EOL goals. Discussion should focus not only on the physical harm but also on the quality of EOL or the dying process.

\section{Consequentialist argument for organ donation}

Informed consent for the DBD or even discussing BD before consenting to organ donation can reduce the success rate of conversion from a potential to actual donor. The best practice is not to ask permission for the DBD. ${ }^{19}$

Evidently, transplantable organs are in serious shortage, and it is paramount to ensure that consenting donors can donate organs. Nevertheless, if withholding informed consent for the DBD is the key to recruiting more donors, the practice is both ethically questionable and potentially manipulative. Such nondisclosure of non-therapeutic intervention falls outside therapeutic privilege. Moreover, this argument lacks convincing empirical evidence. A systematic review showed that as long as the timings of death notification and donation request are 'decoupled', the success rate of the request made before death is the same as or better than that after death. ${ }^{33}$ Instead of hiding consent for the DBD behind the curtain, an ethically correct approach is complete transparency of death determination and the process of organ procurement from the outset.

\section{Consequentialist argument for reducing ICU cost}

Unilateral DBD followed by organ procurement or WLST (in case of non-donor) is the best way to reduce ICU cost. Waiting for the overwhelmed family/surrogate to make a final decision may take a long time and wastes scarce healthcare resources.

The underlying pathology of BD can wax and wane and may slowly evolve over several days. It is not unreasonable to allow 24-48 hours to think and discuss among family members, reflecting on the patient's prestated wishes before choosing an option most consistent with them. As long as a limited time frame is specified, such waiting is not unusual in routine ICU operation and should be accommodated for such critical decisions.

The more problematic situation is the rare case of insisting on indefinite LST without the DBD. Since such a patient is unlikely to be an organ donor, and the longer the patient survives withoutcirculatory collapse the less likely she is truly brain dead, compassionate negotiation by a team of ethics consultants and palliative care specialists to explore a longer term care plan is indicated. The use of a unilateral DBD in such cases for the purpose of terminating care without transparency would only enhance the public distrust that BD is used to cut costs, and lead to the decline of donation rates. Forcing the DBD and urging organ donation after the fact would only harden the resistance from the family, as seen in the aforementioned case of Jahi McMath. ${ }^{3435}$ iv

\section{CONCLUSION}

The majority of deaths in the ICU are after WLST, and among the remaining deaths, depending on the type and location of the ICU, $10 \%-30 \%$ are BD. ${ }^{36-39}$ While WLST is performed under a strict consent process, the DBD has been obtained without informed consent for over half a century, except in a few jurisdictions. The current effort to expand the donor pool seems to be focused on the majority who die after informed consent for WLST. Some authors recommend continuing intensive treatment on cases of catastrophic brain damage with no hope of recovery until full-blown BD develops as opposed to considering WLST. ${ }^{40}$ By not consulting the family/surrogate on this decision, let alone asking for consent, and proceeding from IBD to BD testing by denying a chance for WLST, the number of patients diagnosed with BD would increase and, therefore, the number of potential donors can also increase.

This strategy of deliberately prolonging IBD or non-therapeutic elective ventilation has already been adopted in countries, such as Spain, with an opt-out donation policy. ${ }^{41} 42$ It seems that other countries with opt-in donation policies such as the USA and the UK are trying to adopt this non-therapeutic practice. ${ }^{3}$ While it certainly expands the donor pool, morally, it requires consent from the family/surrogate or through an advance directive. ${ }^{13}$ Full disclosure of this protocol should be presented at the time of informed consent for the DBD.

Certainly, transplantable organs are in dire shortage, but eliminating proper disclosure and consent to start testing for $\mathrm{BD}$ constitutes a serious violation of the principle of respect for autonomy, or, worse, is tantamount to deception. To date, nobody is proposing presumed consent for other newer protocols, such as DCDD (except for certain opt-out countries) and proposed new policies such as donation from donors with higher $\mathrm{BD},{ }^{43}$ the elimination of dead donor rule ${ }^{44}$ and donation after euthanasia. ${ }^{45}$ Without discussing the morality of these newer or proposed protocols to increase transplantable organs, one uncontroversial fact is that they require, as the moral minimum, a strict and explicit informed consent process. I humbly submit here that we make consent for all antemortem, non-therapeutic interventions used for organ procurement explicit, including the procedure of the DBD-the oldest practice among them that began in 1960s, in the era when informed consent was still fledgling.

Acknowledgements I am grateful to two anonymous reviewers, whose penetrating criticisms and insightful comments led to numerous improvements. All remaining faults are, of course, my own responsibility. I also thank Bryan Cwik for giving me the impetus and encouragement to write this essay.

Contributors $\mathrm{OM}$ is the sole author of this article.

Funding The authors have not declared a specific grant for this research from any funding agency in the public, commercial or not-for-profit sectors.

Competing interests None declared.

Patient consent for publication Not required.

Provenance and peer review Not commissioned; externally peer reviewed.

${ }^{\text {iv }}$ This is another reason that the EOL conference proposed here should not be led by an OPO, because the OPO quickly leaves the family who declines donation, and the family is left without advocate. A palliative care specialist can advocate the family regardless of donor status. 
Data availability statement There are no data in this work.

Open access This is an open access article distributed in accordance with the Creative Commons Attribution Non Commercial (CC BY-NC 4.0) license, which permits others to distribute, remix, adapt, build upon this work non-commercially, and license their derivative works on different terms, provided the original work is properly cited, appropriate credit is given, any changes made indicated, and the use is non-commercial. See: http://creativecommons.org/licenses/by-nc/4.0/.

ORCID iD

Osamu Muramoto http://orcid.org/0000-0003-0617-2631

\section{REFERENCES}

1 lacobucci G. Organ donation: England will have "opt-out" system from May 2020. BMJ 2020;368.

2 Register your details - NHS organ donation. Available: https://www.organdonation. nhs.uk/register-your-decision/register-your-details/ [Accessed Mar 2020].

3 Brown S-JR. Organ donor registration in the UK: the need for informed consent for ante-mortem interventions to facilitate organ donation. Med Law Int 2019;19(23):113-35.

4 Lewis A, Bonnie RJ, Pope T, et al. Determination of death by neurologic criteria in the United States: the case for revising the uniform determination of death act. J Law Med Ethics 2019;47(4_suppl):9-24.

5 Veatch RM. Controversies in defining death: a case for choice. Theor Med Bioeth 2019;40(5):381-401.

6 Lewis A. Contentious ethical and legal aspects of determination of brain death. Semin Neurol 2018;38(5):576-82.

7 Yanke G, Rady MY, Verheijde JL. Ethical and legal concerns with Nevada's brain death amendments. J Bioeth Inq 2018:15(2):193-8.

8 Truog RD, Tasker RC. Counterpoint: should informed consent be required for apnea testing in patients with suspected brain death? Yes. Chest 2017;152(4):702-4.

9 Lewis A, Greer D. Point: should informed consent be required for apnea testing in patients with suspected brain death? no. Chest 2017;152(4):700-2.

10 Muramoto 0 . Informed consent for the diagnosis of brain death: a conceptual argument. Philos Ethics Humanit Med 2016;11(1).

11 Shewmon DA. The case of Jahi McMath: a neurologist's view. Hastings Cent Rep 2018:48(Suppl 4):S74-6.

12 Truog RD. Lessons from the case of Jahi McMath. Hastings Cent Rep 2018;48(Suppl 4): $570-3$.

13 Baumann A, Audibert G, Guibet Lafaye C, et al. Elective non-therapeutic intensive care and the four principles of medical ethics. J Med Ethics 2013;39(3):139-42.

14 President's Commission for the Study of Ethical Problems in Medicine and Biomedical and Behavioral Research. Making Health Care Decisions : A report on the ethical and legal implications of informed consent in the patient-practitioner relationship. Washington, D.C: U.S. Government Printing Office, 1982.

15 Schaefer KG, Block SD. Physician communication with families in the ICU: evidencebased strategies for improvement. Curr Opin Crit Care 2009;15(6):569-77.

16 Virdun C, Luckett T, Davidson PM, et al. Dying in the hospital setting: a systematic review of quantitative studies identifying the elements of end-of-life care that patients and their families rank as being most important. Palliat Med 2015;29(9):774-96

17 Kotloff RM, Blosser S, Fulda GJ, et al. Management of the potential organ donor in the ICU: Society of critical care medicine/American College of chest physicians Association of organ procurement organizations consensus statement. Crit Care Med 2015:43(6):1291-325

18 Helms AK, Torbey MT, Hacein-Bey L, et al. Standardized protocols increase organ and tissue donation rates in the neurocritical care unit. Neurology 2004;63(10):1955-7.

19 Lustbader D. Organ donation: practicalities and ethical conundrums. Am J Crit Care 2014;23(1):81-4.
20 Shemie SD, Robertson A, Beitel J, et al. End-Of-Life conversations with families of potential donors: leading practices in offering the opportunity for organ donation. Transplantation 2017;101(5S Suppl 1):S17-26.

21 Uniform Determination of Death Act. 12A U.L.A. 7811980.

22 President's Commission for the Study of Ethical Problems in Medicine and Biomedical and Behavioral Research. Defining death. Washington, D.C.: U.S. Government Printing Office, 1981.

23 Bernat IL, Culver CM, Gert B. On the definition and criterion of death. Ann Intern Med 1981;94(3):389-94.

24 Halevy A, Brody B. Brain death: reconciling definitions, criteria, and tests. Ann Intern Med 1993;119(6):519-25.

25 Lewis A, Adams N, Varelas P, et al. Organ support after death by neurologic criteria: results of a survey of US neurologists. Neurology 2016;87(8):827-34.

26 Daneshmand A, Rabinstein AA, Wijdicks EFM. The apnea test in brain death determination using oxygen diffusion method remains safe. Neurology 2019;92(8):386-7.

27 Wijdicks EFM. The case against confirmatory tests for determining brain death in adults. Neurology 2010;75(1):77-83.

28 Goudreau JL, Wijdicks EF, Emery SF. Complications during apnea testing in the determination of brain death: predisposing factors. Neurology 2000;55(7):1045-8.

29 ASGE Standards of Practice Committee, Fisher DA, Maple JT, et al. Complications of colonoscopy. Gastrointest Endosc 2011;74(4):745-52.

30 Myers J, Arena R, Franklin B, et al. Recommendations for clinical exercise laboratories: a scientific statement from the American heart association. Circulation 2009;119(24):3144-61

31 Nair-Collins M, Miller FG. Commentary: false positives in the diagnosis of brain death. Camb Q Healthc Ethics 2019;28(4):648-56.

32 Joffe AR, Anton NR, Duff JP. The apnea test: rationale, confounders, and criticism. J Child Neurol 2010:25(11):1435-43.

33 Simpkin AL, Robertson LC, Barber VS, et al. Modifiable factors influencing relatives' decision to offer organ donation: systematic review. BMJ 2009;338:b991.

34 Hutchinson D. Family of N.J. girl declared dead files malpractice suit claiming Hospital pressured them for organs. NJ.com, 2015. Available: http://www.nj.com/somerset/ index.ssf/2015/03/family_for_jahi_mcmath_files_lawsuit.html [Accessed March 2020].

35 Bishop JP. Why 'brain death' is contested ground. Harvard Divinity Bulletin 2015:43(1-2). Available: https://bulletin-archive.hds.harvard.edu/articles/ winterspring2015/why-brain-death-contested-ground [Accessed March 2020].

36 Prendergast TJ, Luce JM. Increasing incidence of withholding and withdrawal of life support from the critically ill. Am J Respir Crit Care Med 1997;155(1):15-20.

37 Cook D, Rocker G, Marshall J, et al. Withdrawal of mechanical ventilation in anticipation of death in the intensive care unit. N Engl J Med 2003:349(12):1123-32.

38 Senouci K, Guerrini P, Diene E, et al. A survey on patients admitted in severe coma: implications for brain death identification and organ donation. Intensive Care Med 2004:30(1):38-44

39 Kompanje EJO, Bakker J, Slieker FJA, et al. Organ donations and unused potential donations in traumatic brain injury, subarachnoid haemorrhage and intracerebral haemorrhage. Intensive Care Med 2006;32(2):217-22.

40 de Groot YJ, Jansen NE, Bakker J, et al. Imminent brain death: point of departure for potential heart-beating organ donor recognition. Intensive Care Med 2010:36(9):1488-94.

41 Escudero D, Valentín MO, Escalante JL, et al. Intensive care practices in brain death diagnosis and organ donation. Anaesthesia 2015:70(10):1130-9.

42 Matesanz R, Domínguez-Gil B, Coll E, et al. How Spain reached 40 deceased organ donors per million population. Am J Transplant 2017;17(6):1447-54.

43 Veatch RM. The death of whole-brain death: the plaque of the disaggregators, somaticists, and mentalists. J Med Philos 2005;30(4):353-78.

44 Truog RD, Miller FG, Halpern SD. The dead-donor rule and the future of organ donation. N Eng/ J Med 2013;369(14):1287-9.

45 Bollen J, de Jongh W, Hagenaars J, et al. Organ donation after euthanasia: a Dutch practical manual. Am J Transplant 2016;16(7):1967-72.

46 Wijdicks EFM. Brain death guidelines explained. Semin Neurol 2015;35(2):105-15. 